\title{
A Proposed Architecture for Implementing a Knowledge Management System in the Brazilian National Cancer Institute
}

\author{
José Geraldo Pereira Barbosa * \\ E-mail address: jose.geraldo@estacio.br \\ Mestrado em Administração e Desenvolvimento Empresarial/Universidade Estácio de Sá \\ Rio de Janeiro, RJ, Brazil.
}

\author{
Antônio Augusto Gonçalves \\ E-mail address: augusto@inca.gov.br \\ Instituto Nacional do Câncer - INCA \\ Rio de Janeiro, RJ, Brazil.
}

\section{Vera Simonetti}

E-mail address: vera.simonetti@estacio.br

Mestrado em Administração e Desenvolvimento Empresarial/Universidade Estácio de Sá Rio de Janeiro, RJ, Brazil.

\section{Altino Ribeiro Leitão \\ E-mail address: altino@inca.gov.br Instituto Nacional do Câncer - INCA Rio de Janeiro, RJ, Brazil.}

\begin{abstract}
Because their services are based decisively on the collection, analysis and exchange of clinical information or knowledge, within and across organizational boundaries, knowledge management has exceptional application and importance to health care organizations. This article proposes a conceptual framework for a knowledge management system, which is expected to support both hospitals and the oncology network in Brazil. Under this holistic single-case study, triangulation of multiple sources of data collection was used by means of archival records, documents and participant observation, as two of the authors were serving as INCA staff members, thus gaining access to the event and its documentation and being able to perceive reality from an insider point of view. The benefits derived from the present status of the ongoing implementation, so far, are: (i) speediness of cancer diagnosis and enhanced quality of both diagnosis and data used in epidemiological studies; (ii) reduction in treatment costs; (iii) relief of INCA'S labor shortage; (iii) improved management performance; (iv) better use of installed capacity; (v) easiness of massive (explicit) knowledge transference among the members of the network; and (vi) increase in organizational capacity of knowledge retention (institutionalization of procedures).
\end{abstract}

Key words: knowledge management; information system; health care; hospital management.

Received 26 October 2007; received in revised form 27 February 2009.

Copyright (C) 2009 Brazilian Administration Review. All rights reserved, including rights for translation. Parts of this work may be quoted without prior knowledge on the condition that the source is identified.

* Corresponding author: José Geraldo Pereira Barbosa

Av. Presidente Vargas 642, $22^{\mathrm{O}}$ andar, Rio de Janeiro, RJ, 20071-001, Brazil. 


\section{INTRODUCTION}

Brazil currently has a complex cancer scenario. General incidence and mortality rates are elevated, with the particularly high incidence of prostate cancer in men and breast cancer in women. Theses cases have been responsible for over one hundred thousand deaths per year. There are approximately 480,000 new diagnoses of cancer each year in Brazil, and the vast majority of these patients have had some contact with hospital services. However, research also shows that in several regions of the country long waiting lists for diagnostics and treatments have become commonplace, which leads to a situation of people being diagnosed with cancer at a very advanced stage (National Cancer Institute [INCA], 2008). These sad findings have moved the national health care ministry in recent years to initiate a series of challenging reforms in order to implement early diagnosing clinical procedures. The management of cancer treatment is a long and complex process and the reduction of the patient's waiting time to start cancer treatment plays an increasingly important role. Therefore, any environment focusing on the accessibility to the treatment of a chronic illness like cancer should make every effort to avoid medical errors and fragmentation of care delivery.

The huge number of cancer cases in Brazil means that information is highly sought after by patients and the clinicians involved in their care and those responsible for cancer services, which opens an opportunity window for implementing hospital information systems [HIS]. Hoping to bring together people involved in care planning and delivering - clinicians, managers and patient representatives, the Instituto Nacional de Cancer (National Cancer Institute [INCA]) has been implementing an oncology care network. The network, as conceived by the INCA, is a partnership of both private and public cancer care delivery organizations, whose success will depend heavily on the collection, analysis and exchange of clinical and managerial information or knowledge within and across the partners' organizational boundaries. Its intention is to generate valuable information by answering the requests from patients, government and regulatory bodies with regard to clinical and medical services. Moreover, the integration of key data would help the evaluation of medical procedures and protocols, streamlining the organizational processes and bringing improvements to cancer treatment. Specifically, the network's objectives are as follows: (i) to improve access to information and knowledge at all levels (physicians, hospital administrators, patients); (ii) to create a community of cancer practice knowledge; (iii) to develop an environment of easy and friendly access to relevant information; and (iv) to collaborate with the decision-making process related to cancer care delivery.

In fact, the oncology care network is increasingly becoming a knowledge-based community of health services and patients themselves that share their knowledge, helping reduce administrative bottlenecks and improve the quality of care. As a result, an environment that helps translate information into knowledge is under construction and constitutes, in itself, a driver for quality improvements.

All the above comments bring to mind the need for a knowledge management system that will identify, capture, structure, share and apply an individual's or organization's knowledge, which will result in a competitive advantage and create sources of sustainable development, according to Nonaka and Takeuchi (1995). Therefore, the purpose of this study is to propose a conceptual framework for a knowledge management system in the Brazilian National Cancer Institute. To accomplish this, knowledge management $[\mathrm{KM}]$ enabled health care system is envisioned that will integrate clinical, administrative and financial processes in health care through a common technical architecture, as well as provide a decision support infrastructure for general decision-making. 


\section{KNOWLEDGE MANAGEMENT}

Knowledge management is associated with intellectual capital and the process of creation and diffusion of knowledge embedded in business processes (Wigg, 1993), and constitutes a critical success factor in the current challenging and innovative business scenario. The main premise of knowledge management is the recognition of knowledge as the central point of organizational performance (Drucker, 1993).

In order to enhance organizational performance and create value, knowledge management includes all the processes that deal with the creation, structuring, dissemination and application of knowledge. In the words of Hedlund (1994), these processes can be analyzed at various levels: the individual, group or organization. For Nonaka and Takeuchi (1995), knowledge management is predicated on shared learning, collaboration and the sharing of knowledge at the strategic organizational level. According to Davenport and Prusak (1998), knowledge management not only involves the production of information but also the capture, transmission and analysis of data, as well as the communication of information based on or derived from the data to those who can work on it.

\section{The Nature of Knowledge}

Concerning knowledge itself, there are two main kinds: tacit and explicit. Tacit knowledge is the personal, unarticulated, unexpressed knowledge possessed by an individual. It is the knowledge and expertise that a person has gained over the years through experience, by interacting with others, and through a process of trial and error. This knowledge lies in the individual's brain or in his personal notes, computer files or desk drawers. It has never been completely articulated, recorded, documented or written down in a comprehensive format. Generally found in non-structured form, such as an individual's ideas, insights, values, experiences and judgments, it is more difficult to identify and to manage. Thus, it needs to be structured before it may be stored and processed. On the other hand, knowledge, sometimes, may be explicated, codified and set down in manuals, written procedures, records, notes, graphic representations, audio and visual materials. Stored in databases, explicit knowledge is suitable for access and processing (Nonaka \& Takeuchi, 1995).

Reber, Nonaka and Takeuchi (1992 as cited in Spender, 1996) picture explicit knowledge as the small tip of a huge iceberg of pre-conscious collective knowledge. The major part of it would be formed by tacit knowledge, invisible and completely incorporated into social identity and practice. An individual will understand a message completely only if he understands the body of his organization collective tacit knowledge. In other words, the physical reality is socially built. By similar reasoning, Spender (1996) concludes that interpretation (knowledge) of experience comes from the interaction among the variables that surround the environment and the individual's perception enhanced by experience.

According to Tsoukas (1996), people are co-producers of their own reality, and this will help them to form their attitudes and behaviours. Attitudes are norms and values that the individual perceives as favourable or not, and the behaviours are the expression of these attitudes which can be shown as coherent or incoherent towards its related attitude. Tacit knowledge is one of the main foundations of the individual's attitudes. Therefore, detecting tacit knowledge is complicated by the fact that the individual has the autonomy to decide what and how much should be transmitted to others.

Almost all activities require some combination of explicit and tacit knowledge, and effective knowledge management is the one that captures both of them. In fact, the real challenges to knowledge management lie in being able to identify and capture tacit knowledge so that it can be retrieved when needed. While explicit knowledge is easy to record and transfer, tacit knowledge is difficult to identify, capture, and transmit. Although converting tacit knowledge to explicit knowledge is difficult, it is not impossible. The employee's tacit knowledge is generally transmitted under the form of 
organizational best practices, which are often documented and put into a network, which is subsequently accessed and used by other employees.

For Spender (1996), the big challenge of management is to distinguish the systemic activities that really increase the collective tacit knowledge from those that only generate local and limited knowledge. In the opinion of Hayek (1992 as cited in Spender, 1996), good managers know how to use organizational knowledge efficiently, the kind of knowledge that, although incorporated into people and processes, does not belong totally to any collaborator. So managers must build an organizational space that facilitates the selection of and interaction among different tacit and explicit knowledge available to the organization, both within its internal and external environment.

\section{The Strategic Value of Knowledge}

The health care industry itself is increasingly becoming a knowledge-based community that is connected to hospitals, clinics and patients for sharing knowledge, reducing administrative costs and improving the quality of care. Thus, the success of health care depends critically on the collection, analysis and exchange of clinical information or knowledge within and across organizational boundaries. It is recognized that the spread of new practices is shaped by multiple influences. In this process, physicians play an important role. They can be thought of in terms of very sophisticated knowledge workers (Wickramasinghe, 2000). Like others, (i) physicians "make sense" of this wealth of knowledge (Borghoff \& Pareschi, 1998); (ii) they own the means of production, e. g., their specialized knowledge; (iii) they possess specialized skills and training, which they have acquired by investing significant resources towards their education; and (iv) they make decisions that have farreaching consequences both for their organizations and their patients (Wickramasinghe, 2000).

Thus, it is the interchange of knowledge that represents the significant change in the present way of managing knowledge in comparison to the early days of managing knowledge. It means that an organization's body of knowledge is considered an asset (intellectual capital) only when shared by its employees. Ultimately, this implies seeing the organization as a distributed knowledge system, where managers have to deal with the central question of how to stimulate experts and workers to share their knowledge without facing resistance and insecurity. The main point is how to create knowledge and exchange it among partners. The traditional dichotomy of acquiring information either in reactive mode, to support a specific decision, or in proactive mode, to scan and monitor the environment to detect problems, is not preemptive.

For Grant (1996), the following conditions, when present in the organization, would be primary for transforming knowledge into value: the first is the organizational capacity of disseminating explicit knowledge and of auditing the application of tacit knowledge. The second condition is the capacity of the workforce to fully understand the received knowledge, what is facilitated by a common organizational language. The third factor stems from the organization being able to recognize the collaborator who is responsible for the knowledge creation and to compensate his effort adequately. This is not an easy task because, although incorporated into the workforce, most of the knowledge is generated within the boundaries of the workplace and refer to it specifically. The fourth condition derives from the limited human capacity to acquire, store and process knowledge, the focus of the rationality thesis proposed by Simon, Egidi and Marris (1992). That would require the division of knowledge among specialized organizational areas. And finally, the last condition presumes that knowledge is the critical input of a production system and the one which generates value into products.

Sveiby (2001) conceives the body of intangible assets of an organization as formed by the competence of the professional staff and the internal and external organizational structure. In this author's words, the internal structure is the one filled with management staff and information systems, basically, and its main function is to support the professional staff when delivering services. Secondly, the internal structure, viewed as the conduction wire connecting the organizational body of knowledge, would be used to facilitate the transfer of tacit knowledge among the professional staff. One of the 
most important facets of this process is to provide professionals with the elements for an efficient conversion of knowledge. By conversion of knowledge, Sveiby (1997) understands those activities of (i) gathering information (explicit knowledge) about a potential problem, (ii) applying and transforming it into tacit knowledge, through practice, and finally presenting the problem's solution in an explicit form. It must be kept in mind that this explicit knowledge will be impregnated, in the subtlest ways, by the individuals' attitudes.

\section{INFORMATION SYSTEMS [IS]}

According to Moraes, Silva and Cunha (2004), organizations should make efforts to implement friendly manageable learning environments and to promote learning by doing because these initiatives improve performance without increasing education and training costs. There is a rising recognition that more extensive use of information technologies could do more to improve the performance of health care systems, within the bounds of appropriate measures to protect the confidentiality of private health information. Indeed, the remarkable restructuring of cancer care institutions, from independent local units into regional and national integrated health care delivery organizations, has required a change in the role performed by information systems. On the other hand, it is important to emphasize that, like other complex applications, the design of an IS-based oncology network represents a challenge to health care managers and public authorities.

\section{The Benefits and Limitations}

Fleury and Fleury (2006) argue that knowledge is managed by means of organizational learning processes which can be seen in three dimensions: acquisition and development of knowledge, dissemination of knowledge and organizational memory building. An important point, raised by Fleury and Fleury (2001), is the issue of knowledge transfer from individuals to teams, and from these to the entire organization. According to the authors, while the individual learning process requires from management the comprehension of positive and negative feelings of employees, the team learning process requires the mixing, interpretation and integration of individual knowledge and beliefs into shared collective systems. Regarding the organizational level, individual and team knowledge may be institutionalized in several ways: structure, procedures, rules and symbolic elements. Organizational memory is developed to store and retrieve information, e. g., data related to past experiences, both successful and non-successful, will be easily available to employees. It is not difficult to perceive that both knowledge dissemination and memory building are substantially enhanced by information systems. The centralized databases where knowledge is codified and stored and made available to employees are very effective, especially in the case of explicit knowledge.

The question of information distribution is not a trivial one. Although information technology [IT] tools, such as databases, intranet and e-mail are helpful, Davenport, Harris and Kohli (2001, p. 71) warn that:

...distribution involves more than just how to send knowledge. There is also the question of what kind and how much to send. Several firms try to limit the information and knowledge to those who interact with clients. One firm uses software technologies that filter the knowledge according to a user's predefined categories of importance. The filters select relevant content and distribute the appropriate parts to the appropriate people. Another firm defines what types of information and queries are appropriate for a particular communication method (e-mail, voice-mail). Once it establishes a norm for each communication type, it holds formal training sessions to educate employees on communication protocols and norms.

Terra (2004) shows that advancements in communication technologies and information systems are affecting in a significant way the processes of generation, diffusion and storing of organizational 
knowledge. Based on the results of his research with 428 Brazilian companies, the author argues that in the 166 companies named by him as 'learning companies', one can find (i) information systems that allow efficient communication throughout the whole organization, (ii) employees with wide access to database and organizational knowledge and (iii) incentives for a systematic documentation of organizational knowledge. According to Terra (2004), investments in the infrastructure technology help knowledge management in three aspects:

- Storing of reference materials: codified management can be easily accessed, avoiding efforts duplication;

. Elaboration of expertise maps: database containing descriptions of individual competences inside and outside the organizational space, making the sharing of tacit knowledge easier;

- Just in time knowledge: tools that reduce time and space barriers to knowledge access (videoconference, distance learning etc).

In terms of massive routine and explicit knowledge transfer, Anand, Glick and Manz (2002) point out that IT based systems are the best way of dealing with it. This kind of data transfer is usually necessary to support relatively structured decisions where cause and effect relations are well known. Using the same line of reasoning, Gupta and Govinjaradan (2000, p. 72) affirm "IT is the only viable mechanism to connect efficiently large numbers of geographically dispersed people". According to these authors, intellectual capital is the individual and organizational knowledge stock multiplied by the speed at which it is circulated inside the organization (or network), which highlights the role of IT systems as the speed accelerator.

According to Sveiby (2001), the implementation of Intranet, management information systems and data bases are important initiatives to transfer individual knowledge to the internal structure. On the other hand, specialist systems, such as the cancer diagnosis system of the INCA, improved manmachine interface, simulation environment and interactive learning via Internet help translate organizational learning to the employees.

The process of knowledge conversion, the so called spiral of knowledge in the words of Nonaka and Takeuchi (1995), is comprised of four steps: socialization, externalization, combination and internalization. The combination step is the one dealing with the creation of systemic knowledge by means of new combinations of accumulated knowledge. According these authors, databases and Internet not only help to systematize concepts but also make the flow of new ideas easy.

On the other hand, knowledge management is not merely a question of sophistication of IT infrastructure. Concerning the obstacles to the transfer of best practices (tacit knowledge) among organization co-workers, Szulanski (1996) enumerates the main ones: (i) an inadequate absorption capacity by the knowledge receiver, (ii) lack of knowledge about the production factors involved in the practices, as well as the interaction process among these factors, and (iii) the lack of comprehension, by the receiver, of the organizational context where the practice will be applied, and finally (iv) the lack of empathy between the provider and receiver of knowledge. Indeed, Szulanski (1996) lists several reasons that may make people reluctant to accept a system that encourages knowledge transfer: loss of the power warranted by individual property of specialized knowledge; not being awarded by the transference of knowledge; spending time and energy in transference and the 'non-invented here' (NIH) syndrome that makes people impermeable to knowledge coming from outside their organization. Compounding these problems there is the fact that people will have to acquire new competences to move around the paraphernalia of new IT-based procedures and tools. It is easy to see that those obstacles are beyond IT capability.

Recent research conducted by Meister (2003 as cited in Bertucci, 2005) in 8 private hospitals located in Belo Horizonte, a Brazilian city, shows a low level of research and development [R\&D] activities, intellectual production and training and development [T\&D] activities in the majority of private hospitals. Again, the implementation of IT-based knowledge system may help alleviate some of those problems but will not be a substitute for persistent low funding of research and training. 
It is also important that the manager focuses his attention on knowledge management processes and structures that directly support the strategic initiatives of organizations, such as cancer research, as in the case of the INCA. As Zack (2003, p. 70) argues:

Knowledge management has gotten a bad rap lately, but much of it can be attributed to the fact that most KM initiatives are not focused on strategic knowledge. An organization that defines its strategy in terms of knowledge and identifies the strategic knowledge leverage points will know where to focus its KM efforts, will get a long-term return on its investment, and will best the KM efforts of competitors.

Similarly, Hammer, Leonard and Davenport (2004, p. 17) mention that "technology has immensely improved access to, and transmission of, information, but it cannot create shortcuts to the most valuable kinds of knowledge. That dilemma explains much about why organizations still have trouble managing knowledge". The authors are clearly speaking of individual and collective tacit knowledge.

A final point to be mentioned concerns the obstacles imposed by organizational culture to strategic changes such as the implementation of IT-based knowledge management in the INCA. Mintzberg, Ahlstrand and Lampel (2005) define organizational culture as the body of shared beliefs which are reflected in traditions, habits, stories, symbols, products etc. Acting as a perceptive filter or lens, culture interferes with thinking styles (decision making, analysis procedures etc) of the acculturated people. According to Mintzberg et al. (2005, p. 268), "culture and especially ideology do not encourage strategic change so much as the perpetuation of existing strategy; at best, they tend to promote shifts in position within the organization's overall strategic perspective". Lorsch (1986) argues that introducing and nurturing the values of innovation and flexibility in the organization is an effective way of promoting acceptance of changes. He also mentions that managers should be submitted to a cultural auditing in order to identify their shared beliefs. The author suggests that these shared beliefs must be made visible around the organization as a way to make people aware of their possible prejudices. Naturally, special attention should be paid to the prejudices against IT knowledge management. However, Hernandez and Caldas (2001) warn that some managers sometimes use culture resistance as an excuse for problems arising from ill designed change processes. Contrary to the classical prejudice that human beings are naturally resistant to changes, these authors argue that human beings resist loss but desire change. For the authors, it is important to treat resistance to changes from both collective and individual perspectives. Resistance, depending on several situational and perceptual factors, varies from person to person.

\section{The Foundations of the Proposed Knowledge Management System}

Healthcare organizations generate a massive amount of data, such as electronic medical records, clinical trial data, hospital records and administrative reports, gathered from internal and external sources, such as clinical practices, hospital information systems, and cancer registries. Usually, this huge collection effort is incomplete because data are rarely transformed into a strategic decisionsupport resource. For this purpose, the emergence of knowledge management tools, such as Data Mining [DM], represents an opportunity to convert raw data into knowledge (Cheah \& Abidi, 1999). Knowledge management in healthcare can be regarded as the confluence of concepts and techniques to facilitate the creation, identification, acquisition, development, dissemination and utilization of a healthcare enterprise's knowledge assets (O'Leary, 1998).

The proposed knowledge management system incorporates four steps: creating, structuring, sharing and applying. Figure 1 presents a short list of procedures and tools included in each step. This knowledge process is based on Bose (2003), who describes knowledge as a process that extracts, transforms and disseminates information to be shared and reused by the entire organization. Additionally, the process includes the contribution of Davenport et al. (2001) when he describes the four major goals of the knowledge management systems: to create knowledge replacement, to increase the access of knowledge, to improve the knowledge environment and to manage knowledge as an intangible asset. 
Figure 1: The Knowledge Process

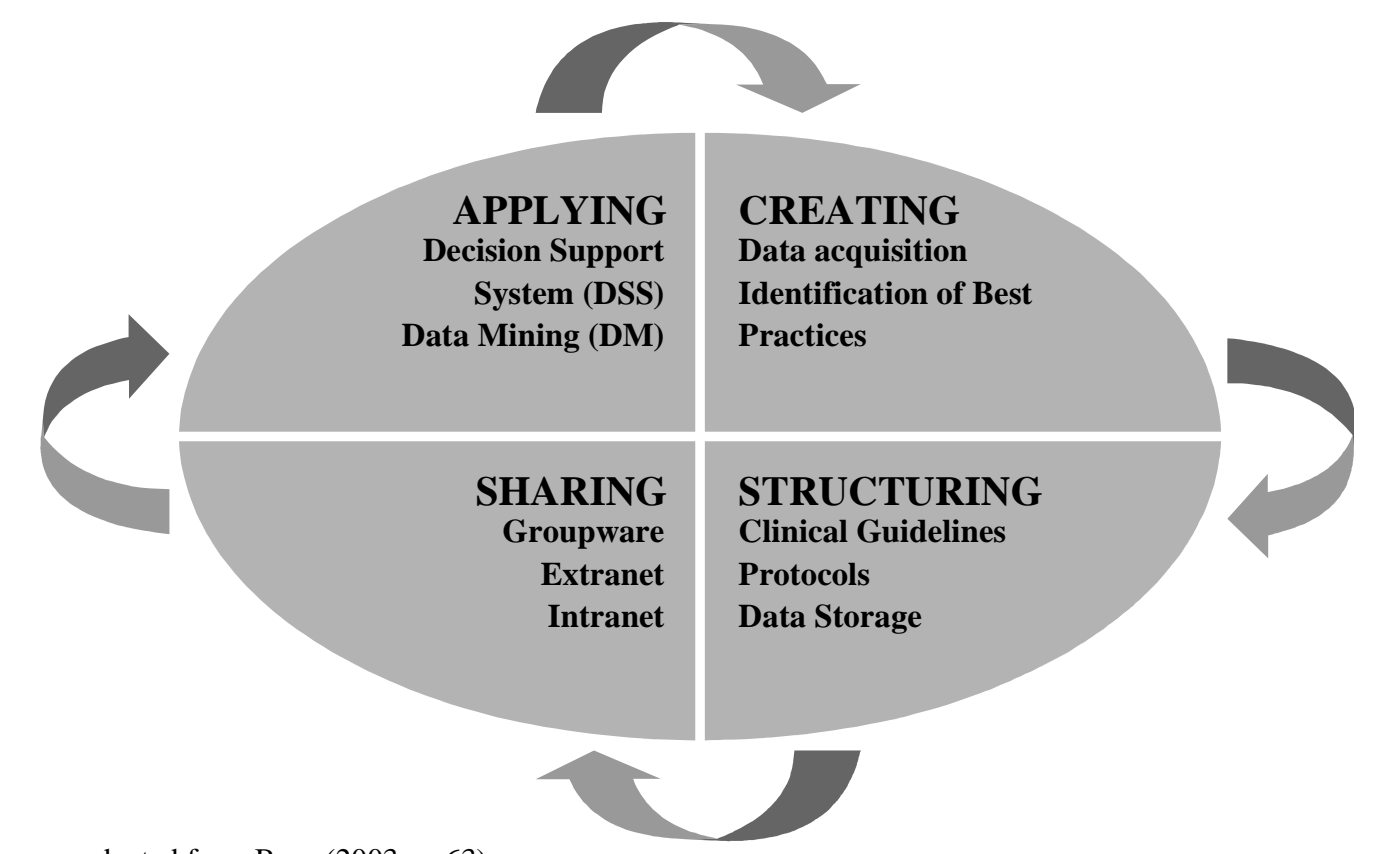

Source: adapted from Bose (2003, p. 63).

The creating step includes knowledge acquisition and knowledge exhibition. As seen before, knowledge comes from different sources, such as clinical learning outcomes, best practices and innovative procedures. The process for acquiring knowledge, from both internal and external sources, is highly dependent on the hospital staff involved in the cancer treatment. Knowledge exhibition is understood to be the formal process representation methods developed by the organization.

The structuring step involves defining, storing, indexing and linking documents and digital images. Mapping existing knowledge, in terms of context and importance, helps classify the knowledge into taxonomies. Storing the knowledge in appropriate repositories such as the yellow pages of expertise, clinical guidelines, protocols and best practices, may then be done.

The sharing step concerns the diffusion of knowledge and collaboration among co-workers, resulting from transfer and dissemination of best practices. Knowledge sharing is accomplished by different means such as training, intranet, groupware, extranets, communities of practice, benchmarking and cross-functional teams.

The last step, applying, involves activities related to decision-making support, problem-solving, developing competency and teamwork, improving productivity, establishing communities of interest, using process workflow, customer support and training to encourage people to speed up the process. 


\section{METHOD}

\section{Research Design}

This study was developed through a qualitative research design to present a descriptive analysis of the conceptual framework of the knowledge management system implemented into the National Cancer Institute (Instituto Nacional de Cancer [INCA]). This system is expected to support both hospitals and the national oncology network, the implementation of which has been placed in the hands of the INCA. The INCA was chosen as a unit of analysis in order to take advantage of the professional experience of two of the authors while working at its Information Technology Division. The first author is the INCA's Chief Information Office [CIO] and associate professor at the graduate program in Business Administration that has been conducting this study. The second and third authors are full professors at the same program while the fourth is the systems manager of the INCA's Information Technology Division.

This holistic single-case study intends to contribute to the knowledge of organizational phenomena, presenting a contemporary description of the system implemented, through an empirical inquiry, answering the questions what, who, where and how (Cooper \& Schindler, 2003; Yin, 2003).

\section{Data Collection}

Triangulation of multiple sources of data collection was used by means of archival records, documents and participant observation, as two authors were serving as INCA staff members, thereby gaining access to the event and its documentation and being able to perceive reality from an insider point of view, characterizing a comprehensive research strategy, in the words of Yin (2003).

The following table summarizes the sourcing procedures:

Table 1: Data Collection Methods

\begin{tabular}{|c|c|}
\hline Source of data & Description \\
\hline Archival Records & $\begin{array}{l}\text { - Clinical data } \\
\text { - Cancer registries } \\
\text { - Patient admission data (demography, rate etc) }\end{array}$ \\
\hline Documents & $\begin{array}{l}\text { - Hospital Information System manuals and user guides; } \\
\text { - Hospital organizational routines (administration, medical care, operations etc) } \\
\text { - Medical research literature }\end{array}$ \\
\hline Interviews & $\begin{array}{l}\text { - } 20 \text { interviews with hospital and administrative staff (nurses, doctors, technicians, } \\
\text { coordinators, managers) }\end{array}$ \\
\hline $\begin{array}{l}\text { Participant } \\
\text { observation }\end{array}$ & $\begin{array}{l}\text { As stated above, two of the researchers (first and fourth) have strong connections with the } \\
\text { problem under investigation, which required careful attention in order to avoid biases. On } \\
\text { the other hand, this fact enabled access to tacit knowledge through the observation of } \\
\text { people directly involved in the problem (physicians, technicians, system analysts etc). }\end{array}$ \\
\hline
\end{tabular}




\section{Data Analysis}

The information was organized in a sequential scheme, under a descriptive approach, crossing information from records and documents with evidence from observation. Following this, the proposed knowledge management system used at the INCA was analyzed through the lens of Bose (2003) and Davenport (2006), Davenport and Prusak (1998), Davenport et al. (2001) and Hammer et al. (2004), seeking converging evidence of their theoretical outlines with the proposed system.

\section{The Proposed ArChitecture}

In most cancer hospitals, multidisciplinary committees discuss their patients' clinical approach. The medical experts go to different sources of information in order to make their decisions. First, they check whether their patients comply with existing guidelines. On the other hand, they can also select their therapeutic decisions based on the cases of patients that have undergone similar treatment in the past.

To support the physicians' activities, several tools, such as tracking mechanisms for keeping the longitudinal patient history, on-line tools for gathering clinical information and the traditional medical record, are used. Most of these are patient-centric and make the hospital environment amenable to the kind of knowledge management system framework, such as the one presented in Figure 2. It can be seen that Figure 2, being based mainly on the recommendations of Bose (2003) and Davenport et al. (2001), incorporates components and associated activities related to all four steps presented in Figure 1.

Figure 2: Proposed Framework for Cancer Knowledge Management

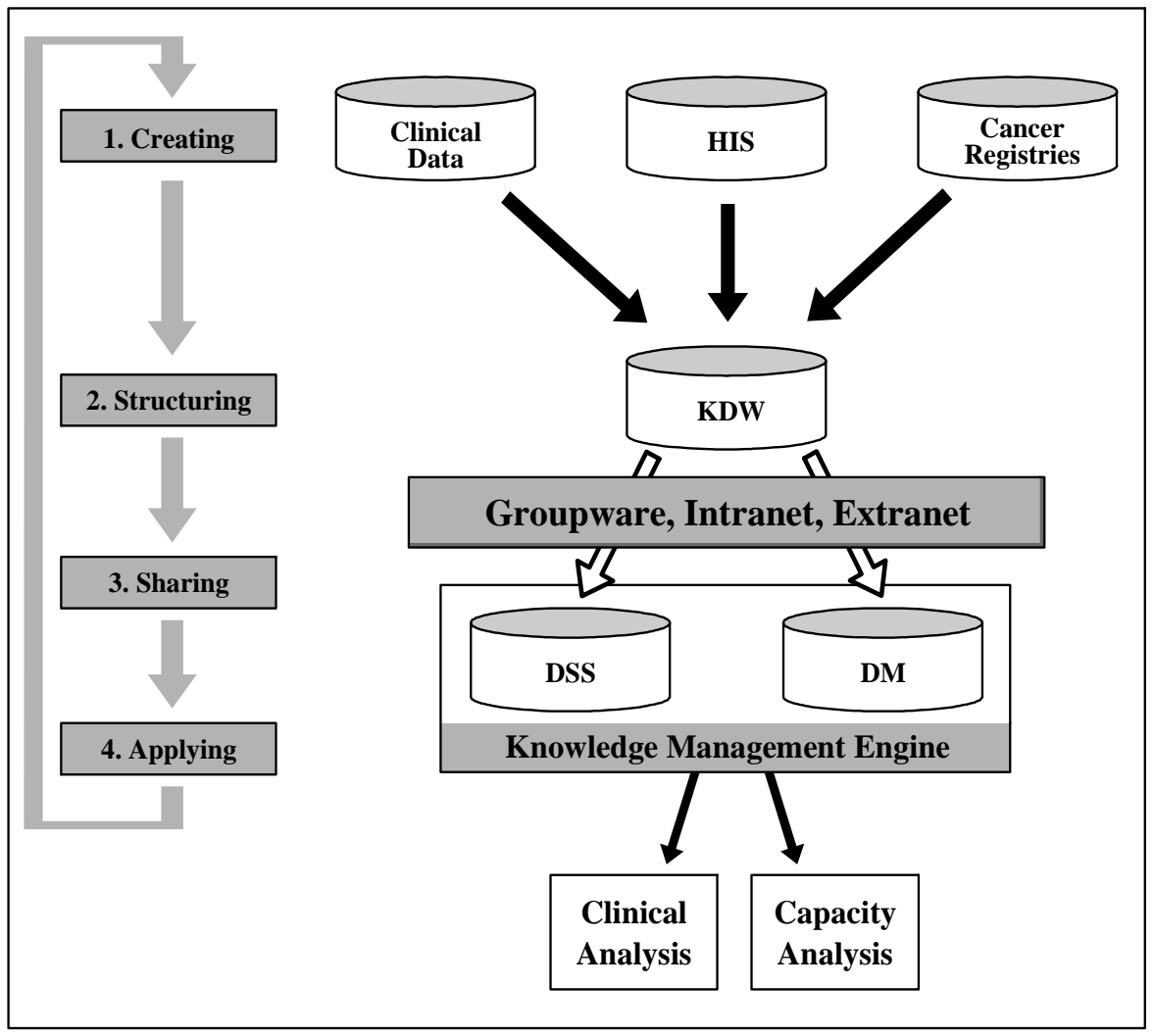

Source: adapted from Bose (2003, p. 68). 


\section{The Main Features of the Proposed Architecture}

\section{Creating}

\section{Clinical Data}

The clinical data, to be incorporated into the Knowledge Data Warehouse [KDW], come from HIS (patients demography) and from physicians, nurses and other health care providers, and fall into three general categories: (i) the historical information that the patient provides; (ii) the information obtained from physical examination; and (iii) the results of the tests or procedures performed on the patient

\section{Hospital Information System [HIS]}

The goal of a hospital information system is to use computers and communications equipment to collect, store, process, retrieve, and communicate, e. g., only administrative information for all hospital activities, and, at the same time, to satisfy the functional requirements of all authorized users. The system is comprised of patient-oriented modules (admission, discharge, order entry, radiology modules and laboratory modules) and administrative modules (finance and billing, management information and decision-support module). In the case of the INCA, Clinical Data is not included in the HIS since this specific module was customized to fit INCA requirements.

\section{Cancer Registries}

The oncology care network aims to develop a national network of cancer registries, which will collect information about patients with cancer from public and private hospitals. Data will be stored and updated to produce a history of all cancer patients, which includes primary treatments, stage of diagnosis, length of survival and subsequent cancers. No matter the patient's status - inpatient, day case or outpatient - cancer registries will collect treatment details. Therefore, it is expected that these registries will play an important role in auditing the quality of cancer services, reducing waiting lists and improving the patient's experience.

\section{Structuring}

\section{Knowledge Data Warehouse}

Data Warehouses act as a repository for current and historical operational data. The health care industry has a poor record in terms of standardization, so data are widely used (and misused) in an ad hoc manner. The knowledge data warehouse [KDW] allows the information to be presented in several formats and to be distributed more widely in communities of practice. At the same time, Online Analytic Processing [OLAP] functionality can be used to gain a deeper understanding of specific health care issues. For Bose (2003), the Knowledge Data Warehouse [KDW] provides the means for business intelligence through ad hoc and managed query environment, OLAP support, statistical analysis tools, knowledge mining and access to Decision Support Systems [DSS] applications.

\section{Intranet, Extranet, Groupware}

By using Intranet/Extranet as a secure access portal, the environment allows for secure, selective sharing of key information, such as test results, follow-up care and support groups. This strategy bridges the gap between what the doctor and the manager knows. The key benefits of e-health strategy adoption and groupware employment are enhanced collaboration between physician and manager, optimization of physicians' work and empowered managers, all of them using sophisticated, costeffective web applications and architecture. According to Bose (2003), executives in leading health care organizations are increasingly recognizing that in order to maintain or gain competitive 
advantages, organizational knowledge not only needs to be managed for, but also integrated with, their corporate systems.

\section{Sharing}

\section{Knowledge Management Engine (DSS and DM)}

The Data Mining [DM] and Decision Support System [DSS] are among the tools used by physicians to gain access to KDW. These tools provide the means for business intelligence through ad hoc and managed query environment, statistical analysis tools, knowledge mining (answers that lead to new questions), and access to decision support software at all hierarchical levels. Goebel and Gruenwald (1999) argue that knowledge-mining tools are used to recognize patterns and relationships that may be valuable for building models that support clinical analysis and capacity analysis. These tools improve the decision-making process by providing new information that otherwise users would not have been able to access on a timely basis. The newly extracted knowledge needs to be inserted into one or more bases to keep them continuously up-to-date and to be of good use for the practice of evidence-based medicine. The data visualization techniques that facilitate the interpretation process of new knowledge can be used in conjunction with the knowledge data warehouse.

Concerning DSS, its most powerful feature is the drill-down capability, which allows users to have access to detailed information, allowing users also to drill up and across. Therefore, the users have unprecedented capabilities to capture, analyze and present data. Physicians, through the experience of using such tools and techniques, gain new knowledge related to their health care area. Specific decision support systems are built using data extracted from various data sources and models.

The data and knowledge necessary for decision-making are spread around the organization. The DSS is programmed to compare the patient's case to the corresponding guideline, then to other cases, and retrieve similar cases. In other words, the system is also designed to be a data warehouse. Thus, the decision-making process itself results in enhanced understanding of the problem process, generating new knowledge, indicating the interdependence between the decision-making and knowledge creation processes.

The Knowledge Management Engine consolidates knowledge from multiple source systems and is capable of presenting different visions of data in order to match the specific requirements of different user segments (e.g. disease, clinical areas, geography). Those visions (data marts) must remain consistent to each other to ensure final report consistency. Furthermore, they must be designed to match the type of analysis required by target users - online analytical processing [OLAP], querying etc. Overall, the access tools provide the means for business intelligence through ad hoc and managed query environment, OLAP support, statistical analysis tools, knowledge mining and access to DSS applications at all levels.

\section{Applying}

\section{Clinical Analysis}

Important beneficiaries of the proposed knowledge management are the activities related to clinical analysis, such as epidemiological and disease analysis and their correspondent management. The frequently mined knowledge required by these activities comes under the form of associations, classes (groups with particular profiles), clusters (groups of instances with the same characteristics), sequences (events linked over a period of time), exceptions (unusual knowledge), forecasts (estimations of future values of attributes), text (e-mails, news) and Web documents. 


\section{Capacity Analysis}

Another beneficiary is the area of capacity analysis that involves comprehensive analysis of patient treatment flow and the availability of capacity (people, machines, etc.) to deal with demand, which often fluctuates. In the course of this kind of analysis, mining of knowledge has frequently been used to help identify patient populations, lab utilization, operating room utilization and patient flow. Davenport (2006) mentions that the knowledge management system is a highly supportive environment for business process analyses.

As seen above, this framework is instrumental to the building of an environment for managing cancer information that incorporates the patient's treatment flow, epidemiological aspects and analysis of installed capacity. These features allow an overview of the national oncology practice which in several ways is much more accurate than viewing each hospital separately.

\section{Current Status of the System Implementation}

In recent years, the INCA has been investing heavily in information technology in order to create favorable conditions for the implementation of the above proposal. A communication infrastructure was established and an information architecture, in which the Hospital Information System [HIS] is one of the most important bases, was implemented. Furthermore, a significant amount of resources has been assigned to the training of managers and operational staff to work in a web environment.

The operational systems, which are data sources to the data warehouse (KDW), have already been implemented. The electronic medical record [EMR] is fully operational in all of the INCA's hospital units and the physicians have been accessing the patient information in real time in the web environment. The Hospital Information System [HIS], the Cancer Registries and Clinical Data are fully implemented. Concerning the Cancer Registries, they have been implemented in more than 100 hospitals around the country and have been generating data to feed the INCA's data warehouse (KDW). Currently in implementation are the Data Mining tools to be used to treat the collected data supporting the epidemiological analysis (clinical and capacity).

\section{FinAL CONSIDERATIONS}

This study presented the capabilities, the technical infrastructure and the decision support architecture to be incorporated into the proposed knowledge management enabled health care management system. The four steps of the framework proposed, i.e., creating, structuring, sharing and applying, represent the pillars of a knowledge structure which could promote a security environment for individuals to express their attitudes through coherent behaviors, facilitating the dissemination of tacit knowledge, issues which have been studied in detail by Davenport (2006), Davenport and Prusak (1998), Davenport et al. (2001) and Hammer et al. (2004).

The benefits derived from the present status of the implementation are reflected mainly on the easiness of knowledge access and on the increase of operational efficiency, not forgetting patient focus and satisfaction, as well as enhanced knowledge transfer and diffusion processes. Specifically, the main results, so far, are:

- Speediness of cancer diagnosis and enhanced quality of both diagnosis and data used in epidemiological studies;

. Reduction in treatment costs;

Relief of the INCA'S labor shortage; 
. Improved management performance;

. Better use of installed capacity;

- Easiness of massive (explicit) knowledge transference if we think of the dozens of medical records daily exchanged among the members of the network; and

. Increase in organizational capacity of knowledge retention (institutionalization of procedures).

The implementation has also demonstrated that a clear understanding of the knowledge management process by professionals and administrative staff is imperative, as mentioned by Lorsch (1986) and Hernandez and Caldas (2001), who also indicate several practical measures to overcome cultural barriers to strategic change. Resistance against the new system on the part of some physicians has been overcome by the development of user-friendly web interfaces, as mentioned by Moraes et al. (2004). An additional facilitator has been the enthusiastic adhesion of young physicians who are used to navigating the web environment.

Above all, the ongoing implementation has revealed that this kind of initiative is most likely to be successful in health care organizations that value organizational learning, pursue strategic goals, nurture a culture of knowledge sharing, accept new challenges, try original approaches and have the ability to exploit the power of information technology. It is not less important to stress that transforming an oncology care network into a knowledge-based community of health services will require the participation of all players: hospitals, clinics, physicians and communities.

Therefore, the sources of data collection used in this study could be strengthened by using semistructured interviews with some staff members, focusing on issues that cannot be examined through the other sources used. Staff participation could provide important insights into the methodology implementation, helping to corroborate previous findings from the other sources through verbal responses.

Finally, Bose (2003) warns that future research on privacy and confidentiality issues of health care knowledge, including the lifetime health care record of patients, is fundamental to the widespread adoption of a health care management system. Significant privacy and confidentiality issues emerge when knowledge from widely disparate sources is brought together and made available in electronic forms.

\section{REFERENCES}

Cheah, Y. N., \& Abidi, S. S. R. (1999). Healthcare knowledge management through building and operationalizing healthcare enterprise memory. In P. Kokol, B. Zupan, J. Stare, M. Premik, \& R. Engelbrecht (Eds.). Medical informatics in Europe (MIE'99) (pp. 726-730). Amsterdam: IOS Press.

Anand, V., Glick, W. H., \& Manz, C. C. (2002). Capital social: explorando a rede de relações da empresa. Revista de Administração de Empresas, 42(4), 57-73.

Bertucci, J. (2005). Ambiente, estratégia e performance organizacional no setor industrial e de serviços. Revista de Administração de Empresas, 45(3), 10-24.

Borghoff, U., \& Pareschi, R. (1998). Information technology for knowledge management. Berlin: Springer-Verlag.

Bose, R. (2003). Knowledge management-enabled health care management systems: capabilities, infrastructure, and decision-support. Expert Systems with Applications, 24(1), 59-71. 
Cooper, D. R., \& Schindler, P. S. (2003). Métodos de pesquisa em administração (7a ed.). Porto Alegre: Bookman.

Davenport, T. H. (2006). Pense fora do quadrado. São Paulo: Elsevier.

Davenport, T. H., \& Prusak, L. (1998). Working knowledge: how organizations manage what they know. Boston: Harvard Business School.

Davenport, T. H., Harri, J. G., \& Kohli, A. K. (2001). How do they know their customers so well? MIT Sloan Management Review, 42(2), 63-73.

Drucker, P. (1993). Post-Capitalist Society. New York: Harper Collins.

Fleury, M. T. L., \& Fleury, A. (2001). Construindo o conceito de competência. Revista de Administração Contemporânea, 5(Edição Especial), 183-196.

Fleury, M. T. L., \& Fleury, A. (2006). Estratégias empresariais e formação de competências (3 ed.). São Paulo: Atlas.

Goebel, M., \& Gruenwald, L. (1999). A survey of data mining and knowledge discovery software tools. ACM SIGKDD, 1(1), 20-33.

Grant, R. M. (1996). Toward a knowledge-based theory of the firm. Strategic Management Journal, 17(Special Issue), 109-122.

Gupta, A. K., \& Govindarajan, V. (2000). Knowledge management's social dimension: lessons from Nucor Steel. Sloan Management Review, 42(1), 71-80.

Hammer, M., Leonard, D., \& Davenport, T. (2004). Why don't we know more about knowledge? MIT Sloan Management Review, 45(4), 13-19.

Hedlund, G. (1994). A model of knowledge management and the n-form corporation. Strategic Management Journal, 15, 73-90.

Hernandez, J. M. C., \& Caldas, M. P. (2001). Resistência à mudança: uma revisão crítica. Revista de Administração de Empresas, 41(2), 31-45.

Lorsch, J. W. (1986). Managing culture: the invisible barrier to strategic change. California Management Journal, 28(2), 95-109.

Mintzberg, H., Ahlstrand, B., \& Lampel, J. (2005). Strategy Safari: a guided tour through the wilds of strategic management. New York: Free Press.

Moraes, L. V. S., Silva, M. A., \& Cunha, C. J. C. A. (2004). A dinâmica da aprendizagem gerencial em um hospital. RAE-eletrônica, 3(2), 1-20. Retrieved October 4, 2007, from http://www.rae.com.br/eletronica/index.cfm?FuseAction=Artigo \&ID=1853\&Secao=ORGANIZ $\mathrm{A} \&$ Volume $=3 \&$ Numero $=2 \& A n o=2004$

National Cancer Institute. (2008). INCA Estimativas 2008: incidência de câncer no Brasil. Rio de Janeiro: Author.

Nonaka, I., \& Takeuchi, H. (1995). The knowledge-creating company. New York: Oxford University Press.

O'Leary, D. (1998). Knowledge management systems: converting and connecting. IEEE Intelligent Systems, 13(3), 30-33.

Simon, H. A., Egidi, M., \& Marris, R. (1992). Economics, bounded rationality, and the cognitive revolution. Aldershot, UK: Elgar. 
Spender, J. C. (1996). Making knowledge the basis of a dynamical theory of the firm. Strategic Management Journal, 17(Special Issue), 45-62.

Sveiby, K. E. (1997). The new organizational wealth. San Francisco: Berrett-Koehler.

Sveiby, K. E. (2001). A knowledge-based theory of the firm to guide in strategy formulation. Journal of Intellectual Capital, 2(4), 344-358.

Szulanski, G. (1996). Exploring internal stickiness: impediments to the transfer of best practice within the firm. Strategic Management Journal, 17(Special Issue), 27-44.

Terra, J. C. C. (2004). Gestão do conhecimento: aspectos conceituais e estudo exploratório sobre as práticas de empresas brasileiras. In M. T. L. Fleury \& M. M. Oliveira Jr. (Orgs.). Gestão estratégica do conhecimento. São Paulo: Atlas.

Tsoukas, H. (1996). The firm as a distributed knowledge system: a constructionist approach. Strategic Management Journal, 17(Special Issue), 11-25.

Wickramasinghe, N. (2000). IS/IT as a tool to achieve goal alignment in the health care industry. International Journal of Healthcare Technology and Management, 2(1), 163-180.

Wigg, K. (1993). Knowledge management foundations. Arlington, VA: Schema.

Yin, R. K. (2003). Case study research: design and methods (3rd ed.). Thousand Oaks, CA: Sage.

Zack, M. H. (2003). Rethinking the knowledge-based organization. MIT Sloan Management Review, 44(4), 66-71. 